\title{
A New Upper Bound on the Infinity Norm of the Inverse of Nekrasov Matrices
}

\section{Lei Gao, Chaoqian Li, and Yaotang Li}

School of Mathematics and Statistics, Yunnan University, Kunming, Yunnan 650091, China

Correspondence should be addressed to Yaotang Li; liyaotang@ynu.edu.cn

Received 1 May 2014; Accepted 24 May 2014; Published 12 June 2014

Academic Editor: Shi-Liang Wu

Copyright (C) 2014 Lei Gao et al. This is an open access article distributed under the Creative Commons Attribution License, which permits unrestricted use, distribution, and reproduction in any medium, provided the original work is properly cited.

A new upper bound which involves a parameter for the infinity norm of the inverse of Nekrasov matrices is given. And we determine the optimal value of the parameter such that the bound improves the results of Kolotilina, 2013. Numerical examples are given to illustrate the corresponding results.

\section{Introduction}

The class of Nekrasov matrices is a subclass of $H$-matrices. Estimating the infinity norm of the inverse of Nekrasov matrices can be used to prove the convergence of matrix splitting and matrix multisplitting iteration methods for solving large sparse systems of linear equations; see [1-4]. Here, we call a matrix $A=\left(a_{i j}\right) \in C^{n, n}$ an $H$-matrix if its comparison matrix $\langle A\rangle=\left[m_{i j}\right]$ defined by

$$
\langle A\rangle=\left[m_{i j}\right] \in C^{n, n}, \quad m_{i j}= \begin{cases}\left|a_{i i}\right|, & i=j \\ -\left|a_{i j}\right|, & i \neq j,\end{cases}
$$

is an $M$-matrix; that is, $\langle A\rangle^{-1} \geq 0[1,5,6]$, and a matrix $A=$ $\left[a_{i j}\right] \in C^{n, n}$ is called a Nekrasov matrix if for each $i \in N$,

$$
\left|a_{i i}\right|>h_{i}(A),
$$

where $h_{1}(A)=\sum_{j \neq 1}\left|a_{1 j}\right|$ and $h_{i}(A)=\sum_{j=1}^{i-1}\left(\left|a_{i j}\right| /\left|a_{j j}\right|\right) h_{j}(A)+$ $\sum_{j=i+1}^{n}\left|a_{i j}\right|, i=2,3, \ldots, n[2,6]$.

In 1975, Varah [7] provided the following upper bound for strictly diagonally dominant (SDD) matrices as one most important subclass of Nekrasov matrices, consequently, $\mathrm{H}$ matrices $[2,6,8]$. Here a matrix $A=\left[a_{i j}\right] \in C^{n, n}$ is called $\mathrm{SDD}$ if for each $i \in N=\{1,2, \ldots, n\}$,

$$
\left|a_{i i}\right|>r_{i}(A) \text {, }
$$

where $r_{i}(A)=\sum_{j \neq i}\left|a_{i j}\right|$.
Theorem 1 (see [7]). Let $A=\left[a_{i j}\right] \in C^{n, n}$ be SDD. Then

$$
\left\|A^{-1}\right\|_{\infty} \leq \frac{1}{\min _{i \in N}\left(\left|a_{i i}\right|-r_{i}(A)\right)} .
$$

We call the bound in Theorem 1 the Varah's bound. As Cvetković et al. [2] said, Varah's bound works only for SDD matrices and even then it is not always good enough. To obtain new upper bounds for the infinity norm of the inverse of a wider class of matrices which sometimes works better in the SDD case, Cvetković et al. [2] give the following bound of Nekrasov matrices.

Theorem 2 (see [2, Theorem 2]). Let $A=\left[a_{i j}\right] \in C^{n, n}$ be $a$ Nekrasov matrix. Then

$$
\begin{aligned}
\left\|A^{-1}\right\|_{\infty} & \leq \frac{\max _{i \in N}\left(z_{i}(A) /\left|a_{i i}\right|\right)}{1-\max _{i \in N}\left(h_{i}(A) /\left|a_{i i}\right|\right)}, \\
\left\|A^{-1}\right\|_{\infty} & \leq \frac{\max _{i \in N} z_{i}(A)}{\min _{i \in N}\left|a_{i i}\right|-h_{i}(A)},
\end{aligned}
$$

where $z_{1}(A)=1$ and $z_{i}(A)=\sum_{j=1}^{i-1}\left(\left|a_{i j}\right| /\left|a_{j j}\right|\right) z_{j}(A)+1, i=$ $2,3 \ldots, n$.

In [9, Theorems 2.2 and 2.3], Kolotilina gave an improvement of these upper bounds in Theorem 2 (see Theorems 3 and 4). 
Theorem 3 (see $\left[9\right.$, Theorem 2.2]). Let $A=\left[a_{i j}\right] \in C^{n, n}$ be $a$ Nekrasov matrix. Then

$$
\left\|A^{-1}\right\|_{\infty} \leq \max _{i \in N} \frac{z_{i}(A)}{\left|a_{i i}\right|-h_{i}(A)} .
$$

Theorem 4 (see $\left[9\right.$, Theorem 2.3]). Let $A=\left[a_{i j}\right] \in C^{n, n}$ be a Nekrasov matrix. Then

$$
\begin{gathered}
\max _{i \in N} \frac{z_{i}(A)}{\left|a_{i i}\right|-h_{i}(A)} \leq \frac{\max _{i \in N}\left(z_{i}(A) /\left|a_{i i}\right|\right)}{1-\max _{i \in N}\left(h_{i}(A) /\left|a_{i i}\right|\right)}, \\
\max _{i \in N} \frac{z_{i}(A)}{\left|a_{i i}\right|-h_{i}(A)} \leq \frac{\max _{i \in N} z_{i}(A)}{\min _{i \in N}\left|a_{i i}\right|-h_{i}(A)} .
\end{gathered}
$$

In this paper, we also focus on the estimation problem of the infinity norm of the inverse of Nekrasov matrices and give an improvement of the bound in Theorem 3 (Theorem 2.2 in [9]). Numerical example is given to illustrate the corresponding results.

\section{Bounds for the Infinity Norm of the Inverse of Nekrasov Matrices}

In order to obtain a new bound, we start with the following lemmas and notations. Given a matrix $A=\left[a_{i j}\right]$, by $A=$ $D-L-U$ we denote the standard splitting of $A$ into its diagonal $(D)$, strictly lower $(-L)$, and strictly upper $(-U)$ triangular parts. And by $[A]_{i j}$ denote the $(i, j)$-entry of $A$; that is, $[A]_{i j}=a_{i j}$. Furthermore, we denote $|A|=\left[\left|a_{i j}\right|\right]$.

Lemma 5 (see [10]). Let $A=\left[a_{i j}\right] \in C^{n, n}$ be a nonsingular $H$-matrix. Then

$$
\left|A^{-1}\right| \leq\langle A\rangle^{-1}
$$

Lemma 6 (see [11]). Given any matrix $A=\left[a_{i j}\right] \in C^{n, n}, n \geq 2$, with $a_{i i} \neq 0$ for all $i \in N$, then

$$
h_{i}(A)=\left|a_{i i}\right|\left[(|D|-|L|)^{-1}|U| e\right]_{i},
$$

where $\in C^{n, n}$ is the vector with all components equal to 1 .

Lemma 7 (see [12]). A matrix $A=\left[a_{i j}\right] \in C^{n, n}, n \geq 2$, is a Nekrasov matrix if and only if

$$
(|D|-|L|)^{-1}|U| e<e,
$$

that is, if and only if $E-(|D|-|L|)^{-1}|U|$ is an SDD matrix, where $E$ is the identity matrix.

Let $C=E-(|D|-|L|)^{-1}|U|=\left[c_{i j}\right]$. Then from Lemma 7, $C$ is SDD when $A$ is a Nekrasov matrix. Note that $c_{11}=1$, $c_{k 1}=0, k=2,3, \ldots, n$, and $c_{1 k}=-\left|a_{1 k}\right| /\left|a_{11}\right|, k=2,3, \ldots, n$, which leads to the following lemma.

Lemma 8. Let $A=\left[a_{i j}\right] \in C^{n, n}$ be a Nekrasov matrix and

$$
C(\mu)=C D(\mu)=\left[E-(|D|-|L|)^{-1}|U|\right] D(\mu),
$$

where $D(\mu)=\operatorname{diag}(\mu, 1, \ldots, 1)$ and $\mu>r_{1}(A) /\left|a_{11}\right|$. Then $C(\mu)$ is $S D D$.
Proof. It is not difficult from (12) to see that $[C(\mu)]_{k 1}=\mu c_{k 1}$ for all $k \in N$ and $[C(\mu)]_{k j}=c_{k j}$ for all $k \in N$ and $j \neq 1$. Hence

$$
[C(\mu)]_{11}=\mu, \quad r_{1}(C(\mu))=r_{1}(C)=\frac{r_{1}(A)}{\left|a_{i i}\right|}
$$

and for $i=2, \ldots, n$,

$$
[C(\mu)]_{i i}=c_{i i}, \quad r_{i}(C(\mu))=r_{i}(C) .
$$

From the fact that $C$ is SDD and $\mu>r_{1}(A) /\left|a_{11}\right|$, we have that $C(\mu)$ is SDD. The proof is completed.

The main result of this paper is the following theorem.

Theorem 9. Let $A=\left[a_{i j}\right] \in C^{n, n}$ be a Nekrasov matrix. Then for $\mu>r_{1}(A) /\left|a_{11}\right|$

$$
\begin{array}{r}
\left\|A^{-1}\right\|_{\infty} \leq \max \{\mu, 1\} \max \left\{\frac{1}{\mu\left|a_{11}\right|-h_{1}(A)},\right. \\
\left.\max _{i \neq 1} \frac{z_{i}(A)}{\left|a_{i i}\right|-h_{i}(A)}\right\} .
\end{array}
$$

Proof. Let $C(\mu)=C D(\mu)=\left(E-(|D|-|L|)^{-1}|U|\right) D(\mu)$, where $D(\mu)=\operatorname{diag}(\mu, 1, \ldots, 1)$. From $(12)$, we have

$$
C(\mu)=(|D|-|L|)^{-1}\langle A\rangle D(\mu)
$$

which implies that

$$
\begin{aligned}
\langle A\rangle & =(|D|-|L|) C(\mu) D(\mu)^{-1} \\
& =(|D|-|L|) \Delta \cdot \Delta^{-1} C(\mu) D(\mu)^{-1},
\end{aligned}
$$

where

$$
\Delta=\operatorname{diag}\left(\delta_{1}, \delta_{2}, \ldots, \delta_{n}\right), \quad \delta_{i}>0, i=1,2, \ldots, n
$$

Furthermore, since a Nekrasov matrix is an $H$-matrix, we have, from Lemma 5,

$$
\begin{gathered}
\left\|A^{-1}\right\|_{\infty} \leq\left\|\langle A\rangle^{-1}\right\|_{\infty} \leq\|D(\mu)\|_{\infty} \cdot\left\|C(\mu)^{-1} \Delta\right\|_{\infty} \\
\cdot\left\|[(|D|-|L|) \Delta]^{-1}\right\|_{\infty} .
\end{gathered}
$$

First, we estimate $\left\|[(|D|-|L|) \Delta]^{-1}\right\|_{\infty}$. Since $(|D|-|L|) \Delta$ is an $M$-matrix and there exists a positive diagonal matrix $\Delta$ such that $(|D|-|L|) \Delta e=e$, see [9], we get

$$
\left\|[(|D|-|L|) \Delta]^{-1}\right\|_{\infty}=\left\|[(|D|-|L|) \Delta]^{-1} e\right\|_{\infty}=1
$$

Secondly, we estimate $\left\|C(\mu)^{-1} \Delta\right\|_{\infty}$. From Lemma $8, C(\mu)$ is SDD. Obviously, multiplying the left-hand side of $C(\mu)$ by diagonal matrix $\Delta^{-1}$ does not change SDD property, 
so $\Delta^{-1} C(\mu)$ is also SDD. Thus, Varah's bound (4) can be applied as follows:

$$
\begin{aligned}
\left\|C(\mu)^{-1} \Delta\right\|_{\infty} & \leq \max _{i \in N} \frac{1}{\left[\Delta^{-1} C(\mu) e\right]_{i}}=\max _{i \in N} \frac{\delta_{i}}{[C(\mu) e]_{i}} \\
& =\max \left\{\frac{\delta_{1}}{[C(\mu) e]_{1}}, \max _{i \neq 1} \frac{\delta_{i}}{[C(\mu) e]_{i}}\right\} \\
& =\max \left\{\frac{\delta_{1}\left|a_{11}\right|}{\mu\left|a_{11}\right|-h_{1}(A)}, \max _{i \neq 1} \frac{\delta_{i}\left|a_{i i}\right|}{\left|a_{i i}\right|-h_{i}(A)}\right\} .
\end{aligned}
$$

In addition, since $z(A)=\left[z_{1}(A), \ldots, z_{n}(A)\right]^{T}=$ $|D|(|D|-|L|)^{-1} e$ and $(|D|-|L|) \Delta e=e$, see $[9,13]$, we have

$$
\delta_{i}\left|a_{i i}\right|=z_{i}(A), \quad i=1,2, \ldots, n .
$$

Substituting (22) into (21), we get that

$$
\left\|C(\mu)^{-1} \Delta\right\|_{\infty} \leq \max \left\{\frac{z_{1}(A)}{\mu\left|a_{11}\right|-h_{1}(A)}, \max _{i \neq 1} \frac{z_{i}(A)}{\left|a_{i i}\right|-h_{i}(A)}\right\} .
$$

Finally, from (20), (23), $z_{1}(A)=1$, and the fact that $\|D(\mu)\|_{\infty}=\max \{\mu, 1\}$, we have

$$
\begin{aligned}
\left\|A^{-1}\right\|_{\infty} \leq & \max \{\mu, 1\} \\
& \times \max \left\{\frac{z_{1}(A)}{\mu\left|a_{11}\right|-h_{1}(A)}, \max _{i \neq 1} \frac{z_{i}(A)}{\left|a_{i i}\right|-h_{i}(A)}\right\} \\
= & \max \{\mu, 1\} \\
& \times \max \left\{\frac{1}{\mu\left|a_{11}\right|-h_{1}(A)}, \max _{i \neq 1} \frac{z_{i}(A)}{\left|a_{i i}\right|-h_{i}(A)}\right\} .
\end{aligned}
$$

The conclusions follow.

Example 10. Consider the Nekrasov matrix $A_{1}$ in $[2,9]$, where

$$
A_{1}=\left[\begin{array}{cccc}
-7 & 1 & -0.2 & 2 \\
7 & 88 & 2 & -3 \\
2 & 0.5 & 13 & -2 \\
0.5 & 3.0 & 1 & 6
\end{array}\right]
$$

By computation, $h_{1}(A)=3.2000, h_{2}(A)=8.2000, h_{3}(A)=$ 2.9609, $h_{4}(A)=0.7359, z_{1}(A)=1, z_{2}(A)=2, z_{3}(A)=$ 1.2971, and $z_{4}(A)=1.2394$. By the bound of Theorem 3 (the bound of Theorem 2.2 in [9]), we have

$$
\left\|A_{1}^{-1}\right\|_{\infty} \leq 0.2632 \text {. }
$$

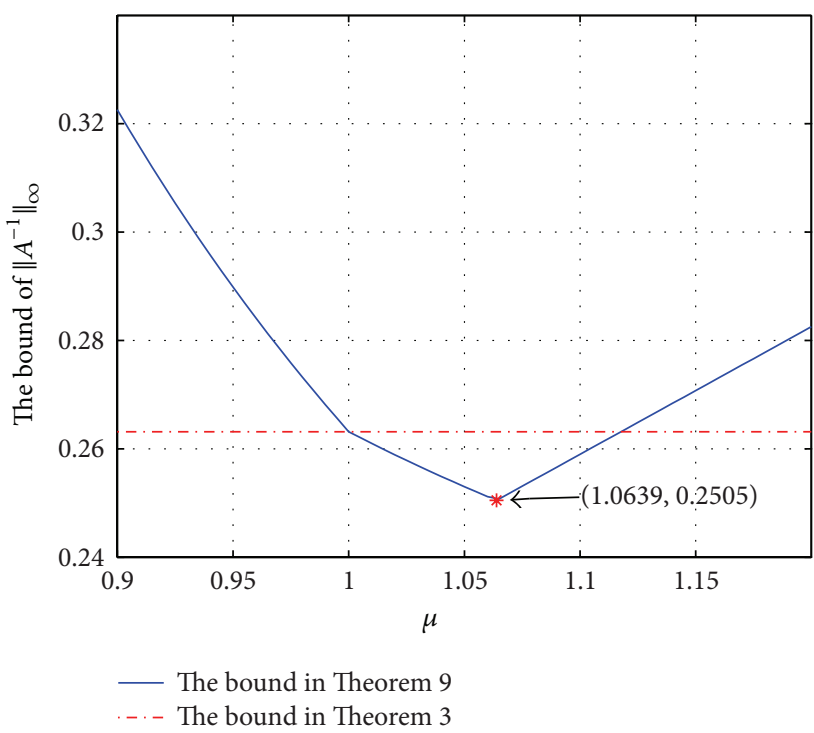

Figure 1: The bounds in Theorems 9 and 3.

By Theorem 9, we have

$$
\begin{aligned}
& \left.\left\|A_{1}^{-1}\right\|_{\infty} \leq 0.3226 \text { (Taking } \mu=0.90\right), \\
& \left.\left\|A_{1}^{-1}\right\|_{\infty} \leq 0.2786 \text { (Taking } \mu=0.97\right), \\
& \left.\left\|A_{1}^{-1}\right\|_{\infty} \leq 0.2549 \text { (Taking } \mu=1.04\right), \\
& \left.\left\|A_{1}^{-1}\right\|_{\infty} \leq 0.2590 \text { (Taking } \mu=1.10\right), \\
& \left.\left\|A_{1}^{-1}\right\|_{\infty} \leq 0.2708 \text { (Taking } \mu=1.15\right) .
\end{aligned}
$$

In fact, $\left\|A_{1}^{-1}\right\|_{\infty}=0.1921$.

Remark 11. Example 10 shows that by choosing the value of $\mu$, the bound in Theorem 9 is better than that in Theorem 3 in some cases. We further observe the bound in Theorem 9 by Figure 1 and find that there is an interval such that for any $\mu$ in this interval, the bound in Theorem 9 for the matrix $A_{1}$ is always smaller than that in Theorem 3. An interesting problem arises: whether there is an interval of $\mu$ such that the bound in Theorem 9 for any Nekrasov matrix is smaller than that in Theorem 3. In the following section, we will study this problem.

\section{The Choice of $\mu$}

In this section, we determine the value of $\mu$ such that the bound for $\left\|A^{-1}\right\|_{\infty}$ in Theorem 9 is less than or equal to that in [9]. First, we consider the Nekrasov matrix $A=\left[a_{i j}\right] \in C^{n, n}$ with

$$
\frac{1}{\left|a_{11}\right|-h_{1}(A)}>\max _{i \neq 1} \frac{z_{i}(A)}{\left|a_{i i}\right|-h_{i}(A)}
$$

and give the following lemma. 
Lemma 12. Let $a, b$, and $c$ be positive real numbers, and $0<$ $a(b-c)<1$. Then

$$
1<\frac{1+a c}{a b}<\frac{1}{a(b-c)} .
$$

Proof. We only need to prove that $(1+a c) / a b-1>0$ and $1 / a(b-c)-(1+a c) / a b>0$. In fact,

$$
\begin{gathered}
\frac{1+a c}{a b}-1=\frac{1-a(b-c)}{a b}>0, \\
\frac{1}{a(b-c)}-\frac{1+a c}{a b}=\frac{c(1-a(b-c))}{a b(b-c)}>0 .
\end{gathered}
$$

The proof is completed.

Lemma 13. Let $A=\left[a_{i j}\right] \in C^{n, n}$ be a Nekrasov matrix with

$$
\frac{1}{\left|a_{11}\right|-h_{1}(A)}>\max _{i \neq 1} \frac{z_{i}(A)}{\left|a_{i i}\right|-h_{i}(A)} \text {. }
$$

Then

$$
\begin{aligned}
1 & <\frac{1+\max _{i \neq 1}\left(z_{i}(A) /\left(\left|a_{i i}\right|-h_{i}(A)\right)\right) \cdot h_{1}(A)}{\left|a_{11}\right| \cdot \max _{i \neq 1}\left((A) /\left(\left|a_{i i}\right|-h_{i}(A)\right)\right)} \\
& <\frac{1 /\left(\left|a_{11}\right|-h_{1}(A)\right)}{\max _{i \neq 1}\left(z_{i}(A) /\left(\left|a_{i i}\right|-h_{i}(A)\right)\right)} .
\end{aligned}
$$

Proof. Let $a=\max _{i \neq 1}\left(z_{i}(A) /\left(\left|a_{i i}\right|-h_{i}(A)\right)\right), b=\left|a_{11}\right|$, and $c=h_{1}(A)$. From (28), we get $0<a(b-c)<1$. Then from Lemma 12, the first and second inequalities in (32) hold.

We now give an interval of $\mu$ such that the bound in Theorem 9 is less than that in Theorem 3.

Lemma 14. Let $A=\left[a_{i j}\right] \in C^{n, n}$ be a Nekrasov matrix with

$$
\frac{1}{\left|a_{11}\right|-h_{1}(A)}>\max _{i \neq 1} \frac{z_{i}(A)}{\left|a_{i i}\right|-h_{i}(A)} \text {. }
$$

Then for each $\mu \in\left(1,\left(1 /\left(\left|a_{11}\right|-h_{1}(A)\right)\right) / \max _{i \neq 1}\left(z_{i}(A) /\left(\left|a_{i i}\right|-\right.\right.\right.$ $\left.\left.h_{i}(A)\right)\right)$ ),

$$
\begin{aligned}
\left\|A^{-1}\right\|_{\infty} \leq & \max \{\mu, 1\} \\
& \times \max \left\{\frac{1}{\mu\left|a_{11}\right|-h_{1}(A)}, \max _{i \neq 1} \frac{z_{i}(A)}{\left|a_{i i}\right|-h_{i}(A)}\right\} \\
< & \max _{i \in N} \frac{z_{i}(A)}{\left|a_{i i}\right|-h_{i}(A)} .
\end{aligned}
$$

Proof. From Lemma 13, we have

$$
\begin{gathered}
\mu \in\left(1, \frac{1+\max _{i \neq 1}\left(z_{i}(A) /\left(\left|a_{i i}\right|-h_{i}(A)\right)\right) \cdot h_{1}(A)}{\left|a_{11}\right| \cdot \max _{i \neq 1}\left(z_{i}(A) /\left(\left|a_{i i}\right|-h_{i}(A)\right)\right)}\right] \\
\bigcup\left[\frac{1+\max _{i \neq 1}\left(z_{i}(A) /\left(\left|a_{i i}\right|-h_{i}(A)\right)\right) \cdot h_{1}(A)}{\left|a_{11}\right| \cdot \max _{i \neq 1}\left(z_{i}(A) /\left(\left|a_{i i}\right|-h_{i}(A)\right)\right)},\right. \\
\left.\frac{1 /\left(\left|a_{11}\right|-h_{1}(A)\right)}{\max _{i \neq 1}\left(z_{i}(A) /\left(\left|a_{i i}\right|-h_{i}(A)\right)\right)}\right)
\end{gathered}
$$

and $\max \{\mu, 1\}=\mu$.
(I) For $\mu \in\left(1,\left(1+\max _{i \neq 1}\left(z_{i}(A) /\left(\left|a_{i i}\right|-h_{i}(A)\right)\right)\right.\right.$. $\left.\left.h_{1}(A)\right) /\left(\left|a_{11}\right| \cdot \max _{i \neq 1}\left(z_{i}(A) /\left(\left|a_{i i}\right|-h_{i}(A)\right)\right)\right)\right]$, then

$$
\mu\left|a_{11}\right|-h_{1}(A) \leq \frac{1}{\max _{i \neq 1}\left(z_{i}(A) /\left(\left|a_{i i}\right|-h_{i}(A)\right)\right)} ;
$$

that is,

$$
\frac{1}{\mu\left|a_{11}\right|-h_{1}(A)} \geq \max _{i \neq 1} \frac{z_{i}(A)}{\left|a_{i i}\right|-h_{i}(A)} .
$$

Therefore,

$$
\begin{aligned}
\max & \{\mu, 1\} \max \left\{\frac{1}{\mu\left|a_{11}\right|-h_{1}(A)}, \max _{i \neq 1} \frac{z_{i}(A)}{\left|a_{i i}\right|-h_{i}(A)}\right\} \\
& =\frac{\mu}{\mu\left|a_{11}\right|-h_{1}(A)} .
\end{aligned}
$$

Consider the function $f(x)=x /\left(x\left|a_{11}\right|-h_{1}(A)\right)$, $x \in\left[1,\left(1+\max _{i \neq 1}\left(z_{i}(A) /\left(\left|a_{i i}\right|-h_{i}(A)\right)\right) \cdot h_{1}(A)\right) /\left(\left|a_{11}\right| \cdot\right.\right.$ $\left.\left.\max _{i \neq 1}\left(z_{i}(A) /\left(\left|a_{i i}\right|-h_{i}(A)\right)\right)\right)\right]$. It is easy to prove that $f(x)$ is a monotonically decreasing function of $x$. Hence, for any $\mu \in\left(1,\left(1+\max _{i \neq 1}\left(z_{i}(A) /\left(\left|a_{i i}\right|-h_{i}(A)\right)\right) \cdot h_{1}(A)\right) /\left(\left|a_{11}\right|\right.\right.$. $\left.\left.\max _{i \neq 1}\left(z_{i}(A) /\left(\left|a_{i i}\right|-h_{i}(A)\right)\right)\right)\right]$,

$$
f(\mu)<f(1) \text {; }
$$

that is,

$$
\frac{\mu}{\mu\left|a_{11}\right|-h_{1}(A)}<\frac{1}{\left|a_{11}\right|-h_{1}(A)}=\max _{i \in N} \frac{z_{i}(A)}{\left|a_{i i}\right|-h_{i}(A)} .
$$

Hence,

$$
\begin{aligned}
\max & \{\mu, 1\} \max \left\{\frac{1}{\mu\left|a_{11}\right|-h_{1}(A)}, \max _{i \neq 1} \frac{z_{i}(A)}{\left|a_{i i}\right|-h_{i}(A)}\right\} \\
& <\max _{i \in N} \frac{z_{i}(A)}{\left|a_{i i}\right|-h_{i}(A)} .
\end{aligned}
$$

(II) For $\in\left[\left(1+\max _{i \neq 1}\left(z_{i}(A) /\left(\left|a_{i i}\right|-h_{i}(A)\right)\right) \cdot h_{1}(A)\right) /\right.$ $\left(\left|a_{11}\right| \cdot \max _{i \neq 1}\left(z_{i}(A) /\left(\left|a_{i i}\right|-h_{i}(A)\right)\right)\right),\left(1 /\left(\left|a_{11}\right|-h_{1}(A)\right)\right) /$ $\left.\max _{i \neq 1}\left(z_{i}(A) /\left(\left|a_{i i}\right|-h_{i}(A)\right)\right)\right)$, then

$$
\mu\left|a_{11}\right|-h_{1}(A) \geq \frac{1}{\max _{i \neq 1}\left(z_{i}(A) /\left(\left|a_{i i}\right|-h_{i}(A)\right)\right)} ;
$$

that is,

$$
\frac{1}{\mu\left|a_{11}\right|-h_{1}(A)} \leq \max _{i \neq 1} \frac{z_{i}(A)}{\left|a_{i i}\right|-h_{i}(A)} .
$$

Therefore,

$$
\begin{aligned}
\max & \{\mu, 1\} \max \left\{\frac{1}{\mu\left|a_{11}\right|-h_{1}(A)}, \max _{i \neq 1} \frac{z_{i}(A)}{\left|a_{i i}\right|-h_{i}(A)}\right\} \\
& =\mu \max _{i \neq 1} \frac{z_{i}(A)}{\left|a_{i i}\right|-h_{i}(A)} .
\end{aligned}
$$


Consider the function

$$
\begin{gathered}
g(x)=x \max _{i \neq 1} \frac{z_{i}(A)}{\left|a_{i i}\right|-h_{i}(A)}, \\
x \in\left[\frac{1+\max _{i \neq 1}\left(z_{i}(A) /\left(\left|a_{i i}\right|-h_{i}(A)\right)\right) \cdot h_{1}(A)}{\left|a_{11}\right| \cdot \max _{i \neq 1}\left(z_{i}(A) /\left(\left|a_{i i}\right|-h_{i}(A)\right)\right)},\right. \\
\left.\frac{1 /\left(\left|a_{11}\right|-h_{1}(A)\right)}{\max _{i \neq 1}\left(z_{i}(A) /\left(\left|a_{i i}\right|-h_{i}(A)\right)\right)}\right] .
\end{gathered}
$$

Obviously, $g(x)$ is a monotonically increasing function of $x$. Hence, for any $\mu \in\left[\left(1+\max _{i \neq 1}\left(z_{i}(A) /\left(\left|a_{i i}\right|-h_{i}(A)\right)\right)\right.\right.$. $\left.h_{1}(A)\right) /\left(\left|a_{11}\right| \cdot \max _{i \neq 1}\left(z_{i}(A) /\left(\left|a_{i i}\right|-h_{i}(A)\right)\right)\right),\left(1 /\left(\left|a_{11}\right|-\right.\right.$ $\left.\left.\left.h_{1}(A)\right)\right) / \max _{i \neq 1}\left(z_{i}(A) /\left(\left|a_{i i}\right|-h_{i}(A)\right)\right)\right)$,

$$
g(\mu)<g\left(\frac{\left(1 /\left(\left|a_{11}\right|-h_{1}(A)\right)\right)}{\max _{i \neq 1}\left(z_{i}(A) /\left(\left|a_{i i}\right|-h_{i}(A)\right)\right)}\right) ;
$$

that is,

$$
\begin{gathered}
\mu \max _{i \neq 1} \frac{z_{i}(A)}{\left|a_{i i}\right|-h_{i}(A)}<\frac{1}{\left|a_{11}\right|-h_{1}(A)} \\
=\max _{i \in N} \frac{z_{i}(A)}{\left|a_{i i}\right|-h_{i}(A)} .
\end{gathered}
$$

Hence,

$$
\begin{aligned}
\max & \{\mu, 1\} \max \left\{\frac{1}{\mu\left|a_{11}\right|-h_{1}(A)}, \max _{i \neq 1} \frac{z_{i}(A)}{\left|a_{i i}\right|-h_{i}(A)}\right\} \\
& <\max _{i \in N} \frac{z_{i}(A)}{\left|a_{i i}\right|-h_{i}(A)} .
\end{aligned}
$$

The conclusion follows from (I) and (II).

Lemma 14 provides an interval of $\mu$ such that the bound in Theorem 9 is better than the bound in Theorem 3 (the bound in [9]). Moreover, we can determine the optimal value of $\mu$ by the following theorem.

Theorem 15. Let $A=\left[a_{i j}\right] \in C^{n, n}$ be a Nekrasov matrix with

$$
\frac{1}{\left|a_{11}\right|-h_{1}(A)}>\max _{i \neq 1} \frac{z_{i}(A)}{\left|a_{i i}\right|-h_{i}(A)} \text {. }
$$

Then

$$
\begin{aligned}
& \min \{\max \{\mu, 1\} \\
& \quad \times \max \left\{\frac{1}{\mu\left|a_{11}\right|-h_{1}(A)}, \max _{i \neq 1} \frac{z_{i}(A)}{\left|a_{i i}\right|-h_{i}(A)}\right\}: \\
&\left.\mu \in\left(1, \frac{1 /\left(\left|a_{11}\right|-h_{1}(A)\right)}{\max _{i \neq 1}\left(z_{i}(A) /\left(\left|a_{i i}\right|-h_{i}(A)\right)\right)}\right)\right\} . \\
&= \frac{z_{1}(A)}{\left|a_{11}\right|}+\max _{i \neq 1} \frac{z_{i}(A)}{\left|a_{i i}\right|-h_{i}(A)} \cdot \frac{h_{1}(A)}{\left|a_{11}\right|} .
\end{aligned}
$$

Furthermore,

$$
\begin{aligned}
\left\|A^{-1}\right\|_{\infty} & \leq \max \{\mu, 1\}\left(\frac{z_{1}(A)}{\left|a_{11}\right|}+\max _{i \neq 1} \frac{z_{i}(A)}{\left|a_{i i}\right|-h_{i}(A)} \cdot \frac{h_{1}(A)}{\left|a_{11}\right|}\right) \\
& <\max _{i \in N} \frac{z_{i}(A)}{\left|a_{i i}\right|-h_{i}(A)} .
\end{aligned}
$$

Proof. From the proof of Lemma 14, we have that

$$
\begin{gathered}
f(x)=\frac{x}{x\left|a_{11}\right|-h_{1}(A)}, \\
x \in\left[1, \frac{1+\max _{i \neq 1}\left(z_{i}(A) /\left(\left|a_{i i}\right|-h_{i}(A)\right)\right) \cdot h_{1}(A)}{\left|a_{11}\right| \cdot \max _{i \neq 1}\left(z_{i}(A) /\left(\left|a_{i i}\right|-h_{i}(A)\right)\right)}\right]
\end{gathered}
$$

is decreasing and that

$$
\begin{gathered}
g(x)=x \max _{i \neq 1} \frac{z_{i}(A)}{\left|a_{i i}\right|-h_{i}(A)}, \\
x \in\left[\frac{1+\max _{i \neq 1}\left(z_{i}(A) /\left(\left|a_{i i}\right|-h_{i}(A)\right)\right) \cdot h_{1}(A)}{\left|a_{11}\right| \cdot \max _{i \neq 1}\left(z_{i}(A) /\left(\left|a_{i i}\right|-h_{i}(A)\right)\right)},\right. \\
\left.\frac{1 /\left(\left|a_{11}\right|-h_{1}(A)\right)}{\max _{i \neq 1}\left(z_{i}(A) /\left(\left|a_{i i}\right|-h_{i}(A)\right)\right)}\right]
\end{gathered}
$$

is increasing. Therefore, the minimum of $f(x)$ and $g(x)$ is

$$
\begin{gathered}
f\left(\frac{1+\max _{i \neq 1}\left(z_{i}(A) /\left(\left|a_{i i}\right|-h_{i}(A)\right)\right) \cdot h_{1}(A)}{\left|a_{11}\right| \cdot \max _{i \neq 1}\left(z_{i}(A) /\left(\left|a_{i i}\right|-h_{i}(A)\right)\right)}\right) \\
\quad=g\left(\frac{1+\max _{i \neq 1}\left(z_{i}(A) /\left(\left|a_{i i}\right|-h_{i}(A)\right)\right) \cdot h_{1}(A)}{\left|a_{11}\right| \cdot \max _{i \neq 1}\left(z_{i}(A) /\left(\left|a_{i i}\right|-h_{i}(A)\right)\right)}\right) \\
=\frac{z_{1}(A)}{\left|a_{11}\right|}+\max _{i \neq 1} \frac{z_{i}(A)}{\left|a_{i i}\right|-h_{i}(A)} \cdot \frac{h_{1}(A)}{\left|a_{11}\right|}
\end{gathered}
$$

which implies that (50) holds. Again by Lemma 14, (51) follows easily.

Remark 16. Theorem 15 provides a method to determine the optimal value of $\mu$ for a Nekrasov matrix $A=\left[a_{i j}\right] \in C^{n, n}$ with

$$
\frac{1}{\left|a_{11}\right|-h_{1}(A)}>\max _{i \neq 1} \frac{z_{i}(A)}{\left|a_{i i}\right|-h_{i}(A)} .
$$

Also consider the matrix $A_{1}$ in Example 10. By computation, we get

$$
\frac{1}{\left|a_{11}\right|-h_{1}(A)}=0.2632>0.2354=\max _{i \neq 1} \frac{z_{i}(A)}{\left|a_{i i}\right|-h_{i}(A)} \text {. }
$$

Hence, by Theorem 15, we can obtain that the bound in Theorem 9 reaches its minimum

$$
\frac{z_{1}(A)}{\left|a_{11}\right|}+\max _{i \neq 1} \frac{z_{i}(A)}{\left|a_{i i}\right|-h_{i}(A)} \cdot \frac{h_{1}(A)}{\left|a_{11}\right|}=0.2505
$$

at $\mu=\left(1+\max _{i \neq 1}\left(z_{i}(A) /\left(\left|a_{i i}\right|-h_{i}(A)\right)\right) \cdot h_{1}(A)\right) /\left(\left|a_{11}\right|\right.$. $\left.\max _{i \neq 1}\left(z_{i}(A) /\left(\left|a_{i i}\right|-h_{i}(A)\right)\right)\right)=1.0639$ (also see Figure 1$)$. 
Next, we study the bound in Theorem 9 for the Nekrasov matrix $A=\left[a_{i j}\right] \in C^{n, n}$ with

$$
\frac{1}{\left|a_{11}\right|-h_{1}(A)} \leq \max _{i \neq 1} \frac{z_{i}(A)}{\left|a_{i i}\right|-h_{i}(A)} .
$$

Theorem 17. Let $A=\left[a_{i j}\right] \in C^{n, n}$ be a Nekrasov matrix with

$$
\frac{1}{\left|a_{11}\right|-h_{1}(A)} \leq \max _{i \neq 1} \frac{z_{i}(A)}{\left|a_{i i}\right|-h_{i}(A)} .
$$

Then we can take $\mu \in\left[\left(1+\max _{i \neq 1}\left(z_{i}(A) /\left(\left|a_{i i}\right|-h_{i}(A)\right)\right)\right.\right.$. $\left.h_{1}(A)\right) /\left(\left|a_{11}\right| \cdot \max _{i \neq 1}\left(z_{i}(A) /\left(\left|a_{i i}\right|-h_{i}(A)\right)\right)\right)$, 1] such that

$$
\begin{aligned}
\left\|A^{-1}\right\|_{\infty} \leq & \max \{\mu, 1\} \\
& \times \max \left\{\frac{1}{\mu\left|a_{11}\right|-h_{1}(A)}, \max _{i \neq 1} \frac{z_{i}(A)}{\left|a_{i i}\right|-h_{i}(A)}\right\} \\
= & \max _{i \in N} \frac{z_{i}(A)}{\left|a_{i i}\right|-h_{i}(A)} .
\end{aligned}
$$

Proof. From (59), we get $\left(1+\max _{i \neq 1}\left(z_{i}(A) /\left(\left|a_{i i}\right|-h_{i}(A)\right)\right)\right.$. $\left.h_{1}(A)\right) /\left(\left|a_{11}\right| \cdot \max _{i \neq 1}\left(z_{i}(A) /\left(\left|a_{i i}\right|-h_{i}(A)\right)\right)\right) \leq 1$. Then, for $\mu>r_{1}(A) /\left|a_{11}\right|=h_{1}(A) /\left|a_{11}\right|$, we have

$$
\begin{aligned}
& \mu \in\left(\frac{h_{1}(A)}{\left|a_{11}\right|}, \frac{1+\max _{i \neq 1}\left(z_{i}(A) /\left(\left|a_{i i}\right|-h_{i}(A)\right)\right) \cdot h_{1}(A)}{\left|a_{11}\right| \cdot \max _{i \neq 1}\left(z_{i}(A) /\left(\left|a_{i i}\right|-h_{i}(A)\right)\right)}\right) \\
& \bigcup\left[\frac{1+\max _{i \neq 1}\left(z_{i}(A) /\left(\left|a_{i i}\right|-h_{i}(A)\right)\right) \cdot h_{1}(A)}{\left|a_{11}\right| \cdot \max _{i \neq 1}\left(z_{i}(A) /\left(\left|a_{i i}\right|-h_{i}(A)\right)\right)}, 1\right] \\
& \bigcup(1,+\infty) .
\end{aligned}
$$

(I) For $\mu \in\left(h_{1}(A) /\left|a_{11}\right|,\left(1+\max _{i \neq 1}\left(z_{i}(A) /\left(\left|a_{i i}\right|-\right.\right.\right.\right.$ $\left.\left.\left.\left.h_{i}(A)\right)\right) \cdot h_{1}(A)\right) /\left(\left|a_{11}\right| \cdot \max _{i \neq 1}\left(z_{i}(A) /\left(\left|a_{i i}\right|-h_{i}(A)\right)\right)\right)\right)$, then $\max \{\mu, 1\}=1$ and

$$
\mu<\frac{1+\max _{i \neq 1}\left(z_{i}(A) /\left(\left|a_{i i}\right|-h_{i}(A)\right)\right) \cdot h_{1}(A)}{\left|a_{11}\right| \cdot \max _{i \neq 1}\left(z_{i}(A) /\left(\left|a_{i i}\right|-h_{i}(A)\right)\right)} ;
$$

that is,

$$
\frac{1}{\mu\left|a_{11}\right|-h_{1}(A)}>\max _{i \neq 1} \frac{z_{i}(A)}{\left|a_{i i}\right|-h_{i}(A)} .
$$

Therefore,

$$
\begin{aligned}
\max & \{\mu, 1\} \max \left\{\frac{1}{\mu\left|a_{11}\right|-h_{1}(A)}, \max _{i \neq 1} \frac{z_{i}(A)}{\left|a_{i i}\right|-h_{i}(A)}\right\} \\
& =\frac{1}{\mu\left|a_{11}\right|-h_{1}(A)} \\
& >\max _{i \neq 1} \frac{z_{i}(A)}{\left|a_{i i}\right|-h_{i}(A)} \\
& =\max _{i \in N} \frac{z_{i}(A)}{\left|a_{i i}\right|-h_{i}(A)} .
\end{aligned}
$$

(II) For $\mu \in\left[\left(1+\max _{i \neq 1}\left(z_{i}(A) /\left(\left|a_{i i}\right|-h_{i}(A)\right)\right) \cdot h_{1}(A)\right) /\left(\left|a_{11}\right| \cdot\right.\right.$ $\left.\left.\max _{i \neq 1}\left(z_{i}(A) /\left(\left|a_{i i}\right|-h_{i}(A)\right)\right)\right), 1\right]$, then $\max \{\mu, 1\}=1$ and

$$
\mu \geq \frac{1+\max _{i \neq 1}\left(z_{i}(A) /\left(\left|a_{i i}\right|-h_{i}(A)\right)\right) \cdot h_{1}(A)}{\left|a_{11}\right| \cdot \max _{i \neq 1}\left(z_{i}(A) /\left(\left|a_{i i}\right|-h_{i}(A)\right)\right)}
$$

that is,

$$
\frac{1}{\mu\left|a_{11}\right|-h_{1}(A)} \leq \max _{i \neq 1} \frac{z_{i}(A)}{\left|a_{i i}\right|-h_{i}(A)} .
$$

Therefore,

$$
\begin{aligned}
\max & \{\mu, 1\} \max \left\{\frac{1}{\mu\left|a_{11}\right|-h_{1}(A)}, \max _{i \neq 1} \frac{z_{i}(A)}{\left|a_{i i}\right|-h_{i}(A)}\right\} \\
& =\max _{i \neq 1} \frac{z_{i}(A)}{\left|a_{i i}\right|-h_{i}(A)} \\
& =\max _{i \in N} \frac{z_{i}(A)}{\left|a_{i i}\right|-h_{i}(A)} .
\end{aligned}
$$

(III) For $\mu \in(1,+\infty)$, then $\max \{\mu, 1\}=\mu$ and

$$
\frac{1}{\mu\left|a_{11}\right|-h_{1}(A)} \leq \max _{i \neq 1} \frac{z_{i}(A)}{\left|a_{i i}\right|-h_{i}(A)} .
$$

Therefore,

$$
\begin{aligned}
\max & \{\mu, 1\} \max \left\{\frac{1}{\mu\left|a_{11}\right|-h_{1}(A)}, \max _{i \neq 1} \frac{z_{i}(A)}{\left|a_{i i}\right|-h_{i}(A)}\right\} \\
& =\mu \cdot \max _{i \neq 1} \frac{z_{i}(A)}{\left|a_{i i}\right|-h_{i}(A)} \\
& >\max _{i \neq 1} \frac{z_{i}(A)}{\left|a_{i i}\right|-h_{i}(A)} \\
& =\max _{i \in N} \frac{z_{i}(A)}{\left|a_{i i}\right|-h_{i}(A)} .
\end{aligned}
$$

The conclusion follows from (I), (II), and (III).

Remark 18. Theorems 15 and 17 provide the value of $\mu$; that is,

$$
\mu=\frac{1+\max _{i \neq 1}\left(z_{i}(A) /\left(\left|a_{i i}\right|-h_{i}(A)\right)\right) \cdot h_{1}(A)}{\left|a_{11}\right| \cdot \max _{i \neq 1}\left(z_{i}(A) /\left(\left|a_{i i}\right|-h_{i}(A)\right)\right)}
$$

such that the bound in Theorem 9 is not worse than that in Theorem 3 for a Nekrasov matrix $A=\left[a_{i j}\right] \in C^{n, n}$. In particular, for the Nekrasov matrix $A$ with $1 /\left(\left|a_{11}\right|-h_{1}(A)\right)>$ $\max _{i \neq 1}\left(z_{i}(A) /\left(\left|a_{i i}-h_{i}(A)\right|\right)\right)$, the bound in Theorem 9 is better than that in Theorem 3 . 
TABLE 1: The upper bounds for $\left\|A_{i}^{-1}\right\|_{\infty}, i=2, \ldots, 6$.

\begin{tabular}{lccccc}
\hline Matrix & $A_{2}$ & $A_{3}$ & $A_{4}$ & $A_{5}$ \\
\hline Exact $\left\|A^{-1}\right\|_{\infty}$ & 0.2390 & 0.8759 & 0.2707 & 1.1519 & - \\
Varah (4) & 1 & 1.4286 & 0.5556 & - & 1.4909 \\
Cvetković et al. (5) & 0.8848 & 1.8076 & 0.6200 & 2.4848 & 1.1557 \\
Cvetković et al. (6) & 0.6885 & 0.9676 & 0.7937 & 1.4138 & 0.5702 \\
Kolotilina (7) & 0.5365 & 0.9676 & 0.5556 & $\mathbf{1 . 4 1 3 8}$ \\
Theorem 9 & $\mathbf{0 . 5 3 6 5}$ & $\mathbf{0 . 9 6 7 6}$ & $\mathbf{0 . 5 0 3 8}$ & $\mathbf{0 . 4 9 2 8}$ \\
\hline
\end{tabular}

Example 19. Consider the following five Nekrasov matrices in $[2,9]$ :

$$
\begin{gathered}
A_{2}=\left[\begin{array}{cccc}
8 & 1 & -0.2 & 3.3 \\
7 & 13 & 2 & -3 \\
-1.3 & 6.7 & 13 & -2 \\
0.5 & 3 & 1 & 6
\end{array}\right], \\
A_{3}=\left[\begin{array}{cccc}
21 & -9.1 & -4.2 & -2.1 \\
-0.7 & 9.1 & -4.2 & -2.1 \\
-0.7 & -0.7 & 4.9 & -2.1 \\
-0.7 & -0.7 & -0.7 & 2.8
\end{array}\right], \\
A_{4}=\left[\begin{array}{cccc}
5 & 1 & 0.2 & 2 \\
1 & 21 & 1 & -3 \\
2 & 0.5 & 6.4 & -2 \\
0.5 & -1 & 1 & 9
\end{array}\right], \quad A_{5}=\left[\begin{array}{ccc}
6 & -3 & -2 \\
-1 & 11 & -8 \\
-7 & -3 & 10
\end{array}\right], \\
A_{6}=\left[\begin{array}{cccc}
8 & -0.5 & -0.5 & -0.5 \\
-9 & 16 & -5 & -5 \\
-6 & -4 & 15 & -3 \\
-4.9 & -0.9 & -0.9 & 6
\end{array}\right] .
\end{gathered}
$$

Obviously, $A_{2}, A_{3}$, and $A_{4}$ are SDD. And it is not difficult to verify that $A_{4}$ satisfies the conditions in Theorem 15 and $A_{2}, A_{3}, A_{5}$, and $A_{6}$ satisfy the conditions in Theorem 17. We compute by Matlab 7.0 the upper bounds for the infinity norm of the inverse of $A_{i}, i=2, \ldots, 6$, which are shown in Table 1. It is easy to see from Table 1 that this example illustrates Theorems 15 and 17.

\section{Conclusions}

In this paper, we give an improvement on the infinity norm bound for the inverse of a Nekrasov matrix in [9]. In particular, for the Nekrasov matrix $A=\left[a_{i j}\right] \in C^{n, n}$ with

$$
\frac{1}{\left|a_{11}\right|-h_{1}(A)}>\max _{i \neq 1} \frac{z_{i}(A)}{\left|a_{i i}\right|-h_{i}(A)},
$$

we prove that new bound is better than that in [9]. However, for the Nekrasov matrix $A$ with

$$
\frac{1}{\left|a_{11}\right|-h_{1}(A)} \leq \max _{i \neq 1} \frac{z_{i}(A)}{\left|a_{i i}\right|-h_{i}(A)},
$$

we only obtain that new bound is equal to that in [9]. For this case, we try to found some better bounds in future. On the other hand, our bound only considers one parameter $\mu$, that is, $D(\mu)=\operatorname{diag}(\mu, 1, \ldots, 1)$, which poses an interesting problem: whether we further improve this bound by introducing more parameters. In future, we will research this problem.

\section{Conflict of Interests}

The authors declare that there is no conflict of interests regarding the publication of this paper.

\section{Acknowledgments}

This research is supported by the National Natural Science Foundation of China (11361074 and 11326242), the Science Foundation of the Education Department of Yunnan Province of China (2013FD002), and Science and Technology Innovation Fund projects of Yunnan University (ynuy201366).

\section{References}

[1] Z.-Z. Bai and D.-R. Wang, "Generalized matrix multisplitting relaxation methods and their convergence," Numerical Mathematics. A Journal of Chinese Universities. (English Series), vol. 2, no. 1, pp. 87-100, 1993.

[2] L. Cvetković, P.-F. Dai, K. Doroslovački, and Y.-T. Li, "Infinity norm bounds for the inverse of Nekrasov matrices," Applied Mathematics and Computation, vol. 219, no. 10, pp. 5020-5024, 2013.

[3] J. G. Hu, "Estimates of $\left\|B^{-1} A\right\|_{\infty}$ and their applications," Mathematica Numerica Sinica. Jisuan Shuxue, vol. 4, no. 3, pp. 272-282, 1982.

[4] J. G. Hu, "Scaling transformation and convergence of splittings of a matrix," Mathematica Numerica Sinica, vol. 5, no. 1, pp. 7278, 1983.

[5] L. Cvetković, "H-matrix theory vs. eigenvalue localization," Numerical Algorithms, vol. 42, no. 3-4, pp. 229-245, 2006.

[6] L. Cvetković, V. Kostić, and K. Doroslovački, "Max-norm bounds for the inverse of S-Nekrasov matrices," Applied Mathematics and Computation, vol. 218, no. 18, pp. 9498-9503, 2012.

[7] J. M. Varah, "A lower bound for the smallest singular value of a matrix," Linear Algebra and Its Applications, vol. 11, pp. 3-5, 1975.

[8] W. Li, "On Nekrasov matrices," Linear Algebra and Its Applications, vol. 281, no. 1-3, pp. 87-96, 1998.

[9] L. Y. Kolotilina, "On bounding inverse to Nekrasov matrices in the infinity norm," Zapiski Nauchnykh Seminarov POMI, vol. 419, pp. 111-120, 2013. 
[10] A. Berman and R. J. Plemmons, Nonnegative Matrices in the Mathematical Sciences, Academic Press, New York, NY, USA, 1979.

[11] F. Robert, "Blocs-H-matrices et convergence des méthodes itératives classiques par blocs," Linear Algebra and Its Applications, vol. 2, pp. 223-265, 1969.

[12] T. Szulc, "Some remarks on a theorem of Gudkov," Linear Algebra and its Applications, vol. 225, pp. 221-235, 1995.

[13] V. V. Gudkov, "On a certain test for non-singularity of matrices," in Latv. Mat. Ezhegodnik (1965), pp. 385-390, Zinatne, Riga, Latvia, 1966. 


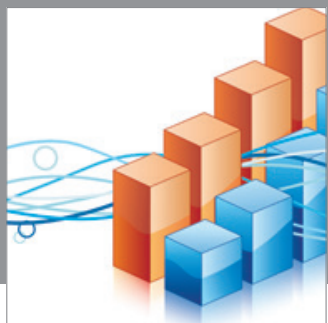

Advances in

Operations Research

mansans

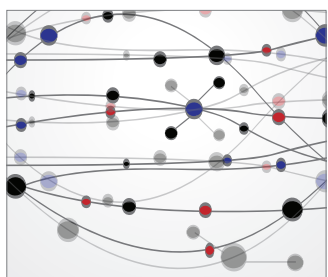

The Scientific World Journal
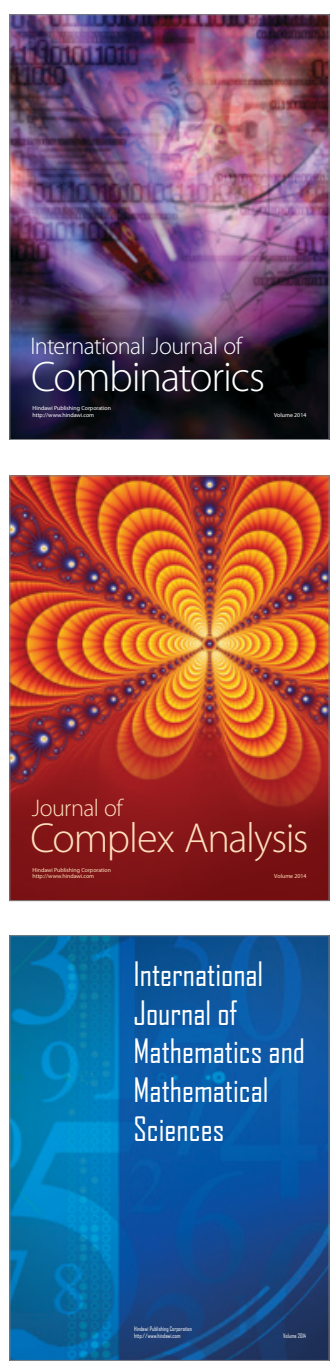
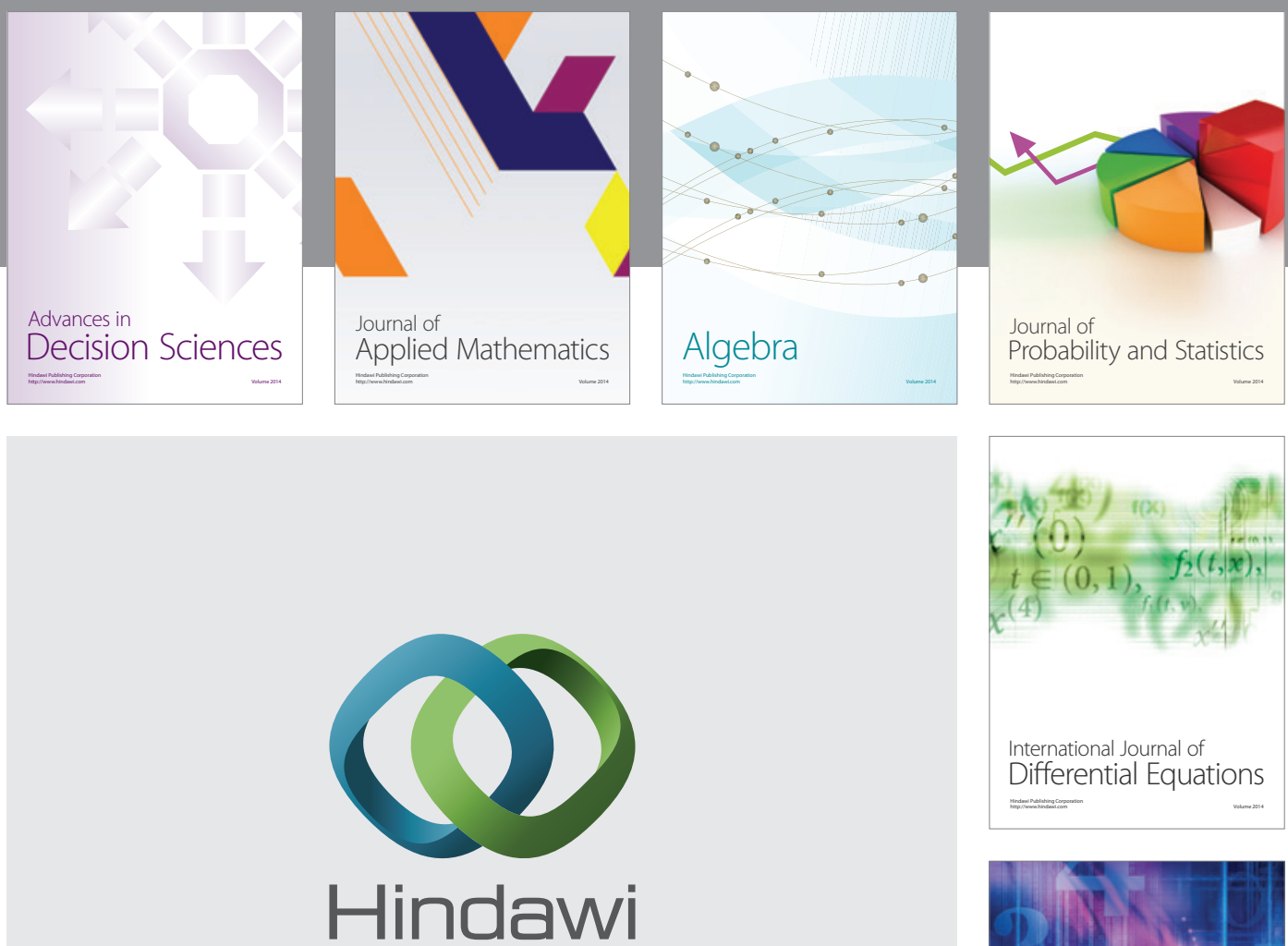

Submit your manuscripts at http://www.hindawi.com
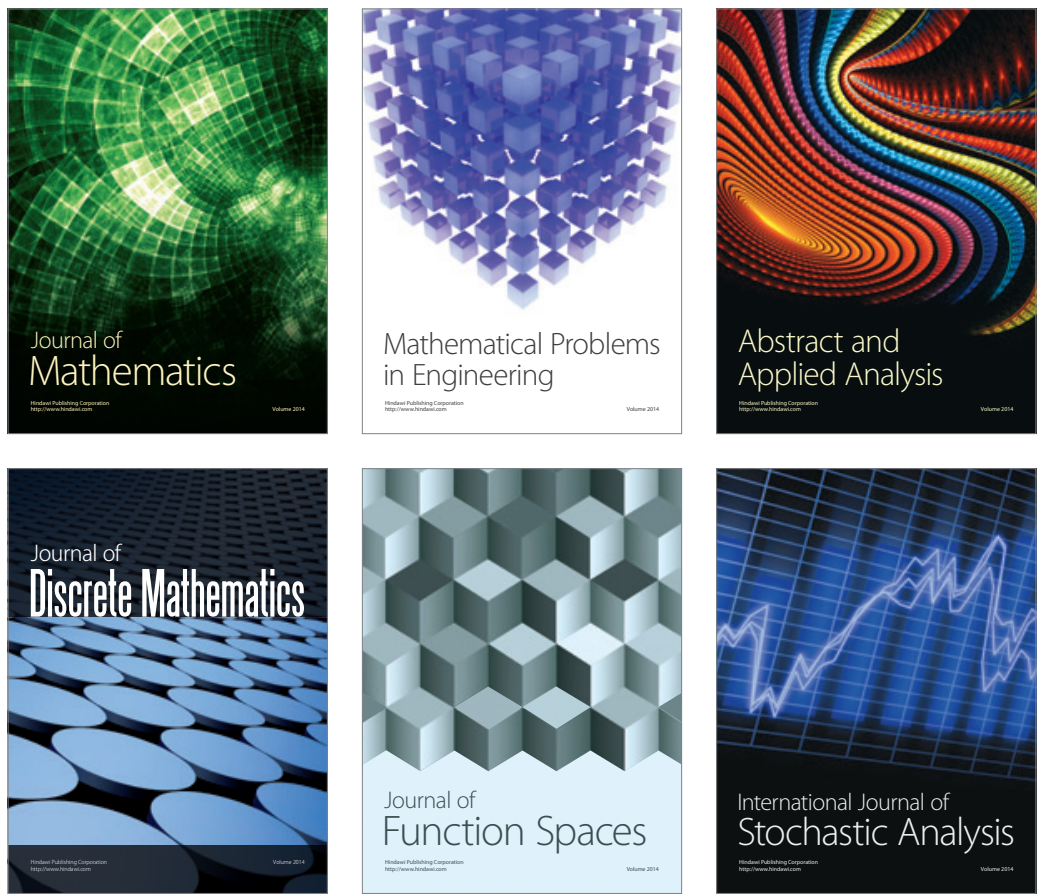

Journal of

Function Spaces

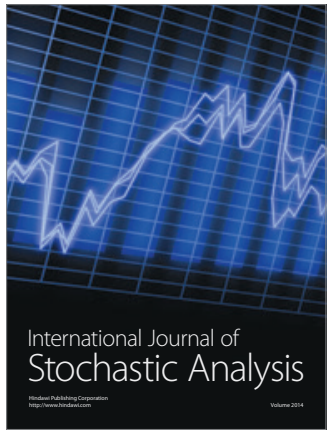

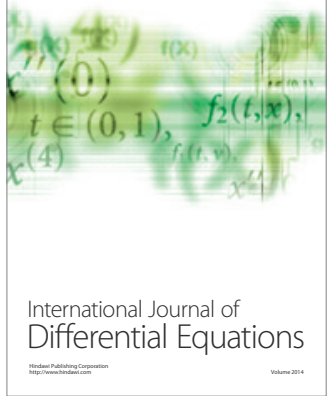
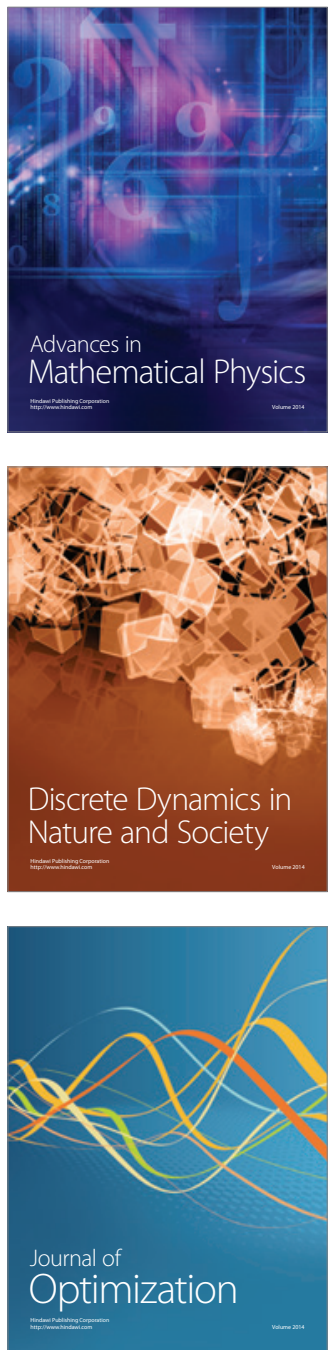